\title{
Orofacial Soft Tissue Reconstruction with Locoregional Flaps in a Health Resource-Depleted Environment: Experiences from Nigeria
}

\author{
Rowland Agbara ${ }^{1}$, Athanasius Chukwudi Obiadazie ${ }^{2}$, Benjamin Fomete ${ }^{2}$, Kelvin Uchenna Omeje ${ }^{3}$ \\ ${ }^{1}$ Oral and Maxillofacial Surgery Unit, Dental and Maxillofacial Surgery Department, Jos University Teaching Hospital, Jos; ${ }^{2}$ Oral and \\ Maxillofacial Surgery Department, Ahmadu Bello University Teaching Hospital, Shika-Zaria; ${ }^{3}$ Oral and Maxillofacial Surgery Unit, Dental \\ and Maxillofacial Surgery Department, Aminu Kano Teaching Hospital, Kano State, Nigeria
}

Background Reconstruction of orofacial soft tissue defects is often challenging due to functional and aesthetic demands. Despite advances in orofacial soft tissue defect reconstruction using free flaps, locoregional flaps still remain an important option, especially in health resource-depleted environments. This retrospective study highlights our experiences in oral and maxillofacial soft tissue reconstruction using locoregional flaps.

Methods A twenty-three years retrospective analysis of all patients managed in our department was undertaken. Information was sourced from patients' case notes and operating theater records. Data was analyzed using SPSS ver. 16 (SPSS Inc.) and Microsoft Excel 2007 (Microsoft).

Results A total of 77 patients underwent orofacial soft tissue defect reconstruction within the years reviewed. Males accounted for $55(71.4 \%)$ cases and trauma was the main etiological factor in $45(58.4 \%)$ of the patients treated. When sites of defect were considered, the lip, 27 (32.1\%), was the most frequent site followed by the nose, 17 (20.2\%). Forehead flap, $51(59.3 \%)$, was the most commonly used flap. Complications noted were tumor recurrences at the recipient bed in $3(3.9 \%)$ cases, tumor occurrence at the donor site in $1(1.3 \%)$ case and postoperative infection in $11(14.3 \%)$ cases.

Conclusions Locoregional flaps still have an important role in the rehabilitation of patients with orofacial soft tissue defects. They remain a vital tool in the armamentarium of the reconstructive surgeon, especially in health resource-depleted environments where advanced reconstructive techniques may not be feasible.

Keywords Health resources / Soft tissue injuries / Face / Surgical flaps
Correspondence: Rowland Agbara Oral and Maxillofacial Surgery Unit, Dental and Maxillofacial Surgery Department, Jos University Teaching Hospital, Jos, Nigeria Tel: +234-803-462-7811 E-mail: row_prof@yahoo.com

No potential conflict of interest relevant to this article was reported.

Received: 24 Oct $2015 \bullet$ Revised: 22 Feb $2016 \bullet$ Accepted: 16 Mar 2016

pISSN: 2234-6163 • elSSN: 2234-6171 • http://dx.doi.org/10.5999/aps.2016.43.3.265・Arch Plast Surg 2016;43:265-271

\section{INTRODUCTION}

The soft tissues in the oral and maxillofacial region (OMFR) such as the eyelids, nose, lips, cheek, and tongue contribute to important functions, which include vision, respiration, mastica- tion, swallowing, and speech. In addition, they contribute significantly to the perception of the beauty of an individual. Since individuals place a high value on facial aesthetics, soft tissue defects in the OMFR may negatively affect the perception of facial beauty, resulting in significant psychological morbidity in addi- 
tion to functional problems [1].

Soft tissue defects in the OMFR may be congenital (as in cleft lip and palate), or acquired. Acquired defects are usually more common, and these may result from trauma, surgery, or infections. Over the years, reconstruction of orofacial soft tissue defects has advanced considerably. Traditionally, the reconstructive ladder approach has been advocated in soft tissue defect reconstruction, and this allows a stepwise option from the simplest to the most complex procedures. These options include healing by secondary intention, primary closure, skin grafting, use of locoregional flaps (with or without tissue expansion), and free flap transfer. Recently, however, the reconstructive escalator or elevator approach has been advocated, because reconstruction should be individualized to each patient and not based on a rigid, stepwise approach [2]. The use of orofacial prostheses has also contributed significantly to the restoration of an acceptable functional and aesthetic status for patients following soft tissue defect [3]. Although each of these reconstructive options achieves different degrees of functional, aesthetic, and psychological rehabilitation for patients, they have various advantages and disadvantages. Skin grafting is a relatively easy procedure, but its disadvantages include poor color match, textural and thickness differences with recipient bed tissues, pigmentation (hypopigmentation and hyperpigmentation), and skin graft contraction $[4,5]$. Tissue expansion has the advantage of good color and texture match, preservation of hair-bearing quality, and increased vascularity with greater length of survival for random flaps elevated in expanded skin. However, its drawbacks include frequent hospital visits for inflation, discomfort, a period of increased deformity during the time of inflation, and the prolonged length of time it takes to expand the adjacent tissue $[6,7]$. Free flaps provide abundant tissues for three-dimensional reconstruction, allow for a single-stage procedure, are more resistant to radiation (which is important in head-and neck-cancer patients requiring radiotherapy), and provide greater freedom to orient and shape the flap [8]. However, free flaps are technique-sensitive, usually require good blood vessels at the recipient site, increase operation time, require extensive postoperative monitoring, and may be contraindicated in some patients with co-morbid conditions $[9,10]$. Locoregional flaps reduce vulnerability to infection and thrombosis, are easier to raise and transfer compared to free flaps, and can provide excellent color match. Limited reach of locoregional flaps, difficulty in achieving three-dimensional reconstruction or coverage of extensive tissue defects, and occasional need for a multistage procedure are some of their limitations [11]. Moreover, locoregional flaps frequently have complications in irradiated fields and may require specific patient positioning to raise [9].
In current practice, locoregional flaps still play a vital role in the reconstruction of head and neck soft tissue defects. They are the major option for head and neck reconstruction in environments where microvascular free tissue transfer is not feasible. In technologically developed environments, locoregional flaps are used as rescue flaps following free flap failure, as well as in patients with relative contraindications to free flap transfer such as the presence of co-morbid medical conditions [10].

In our environment, health care delivery still faces many challenges, and these have significantly affected the scope of practice relative to developed societies. Some of these challenges include scarce health resources/infrastructure, low government expenditure on health, limited overseas exposure in areas of need for capacity building, and lack of quality assurance programs [12]. Free flap transfer is technique-sensitive and therefore requires the availability of infrastructure and high-level health personnel resources to achieve success and reduce avoidable mistakes that could lead to fatalities. The absence of these critical elements has made free tissue transfer difficult to achieve in this environment presently: hence our reliance on locoregional flaps. The aim of our study, therefore, is to review our experiences with locoregional flaps in the reconstruction of soft tissue defects of the OMFR.

\section{METHODS}

All patients with soft tissue defect in the OMFR reconstructed using locoregional flaps at a regional university teaching hospital between April 1991 and May 2014 were retrospectively studied. Information was sourced from patients' case notes and the operating theater register. Information retrieved included age, sex, etiology of orofacial defects, indication for surgical reconstruction, types of locoregional flap, timing of flap division, duration of hospital stay, and complications.

Various locoregional flaps were used for the reconstruction of the diverse OMFR defects. Among them, forehead flaps were most commonly used. The forehead flap was classified as complete if the whole forehead tissue between hairline and supraorbital rim was mobilized (from a point perpendicular to the lateral canthal region on one side to a corresponding point or beyond on the contralateral side), and partial, if only a part of the forehead tissue was mobilized. Data retrieved was analyzed using SPSS ver. 16 (SPSS Inc., Chicago, IL, USA) and Microsoft Office Excel 2007 (Microsoft, Redmond, WA, USA). Findings from descriptive statistics were represented in the form of graphs, tables, and charts with statistical significance tested using Pearson's chi-square $\left(\chi^{2}\right)$ and set at $\mathrm{P}<0.05$. 


\section{RESULTS}

A total of 77 patients underwent oral and maxillofacial soft tissue defect reconstruction using locoregional flaps within the years reviewed, and this consisted of 55 (71.4\%) males and 22 (28.6\%) females, giving a male to female ratio of 2.5:1. The age of the patients ranged from 4 to 76 years with a mean of $33.4 \pm$ 17.0 years. A high number of patients fell into one of three age groups: $10-19$ (19.5\%), 20-29 (19.5\%), and 40-49 (22.1\%) years (Table 1). Trauma, in 45 (58.4\%) patients, was the most common etiological factor, followed by tumor excision, 18 (23.4\%) patients (Fig. 1). Of the trauma-associated soft tissue defects, assault, 17 (37.8\%) patients, accounted for the highest number of cases, while malignant tumor excision accounted for 17 (94.4\%) of the 18 surgical defects associated with tumor excision. Soft tissue defects arising from infection, 11 (14.3\%) patients, were mainly due to orofacial gangrene (cancrum oris, nasalis, and oculi).

Table 1. Patients who underwent locoregional flap reconstruction grouped by age

\begin{tabular}{|lc|}
\hline Age group (yr) & Number $(\%)$ \\
\hline $0-9$ & $5(6.5)$ \\
$10-19$ & $15(19.5)$ \\
$20-29$ & $15(19.5)$ \\
$30-39$ & $11(14.3)$ \\
$40-49$ & $17(22.1)$ \\
$50-59$ & $8(10.4)$ \\
$60-69$ & $3(3.9)$ \\
$70-79$ & $3(3.9)$ \\
Total & $77(100.0)$ \\
\hline
\end{tabular}

General anesthesia was used in $76(98.7 \%)$ patients, while local anesthesia with conscious sedation was used in $1(1.3 \%)$ patient. The lip, 27 (32.1\%) patients, was the most common site of defect location, followed by the nose, 17 (20.2\%) patients, and cheek, 16 (19.0\%) patients (Table 2). Although males dominated in all the sites analyzed for orofacial defect, this was not statistically significant $(\mathrm{P}=0.485)$. Forehead flap, $51(59.3 \%)$ patients, was the most frequently used locoregional flap (Table 2) and consisted of 32 complete and 19 partial forehead flaps. Of the 77 patients studied, the timing of flap raising was documented in only 67 (87.0\%) patients. A higher number of patients (57 cases) had delayed reconstruction, while 10 patients

\section{Fig. 1. Etiology of orofacial defects reconstructed}

Frequency of reconstructed orofacial defects by etiology.

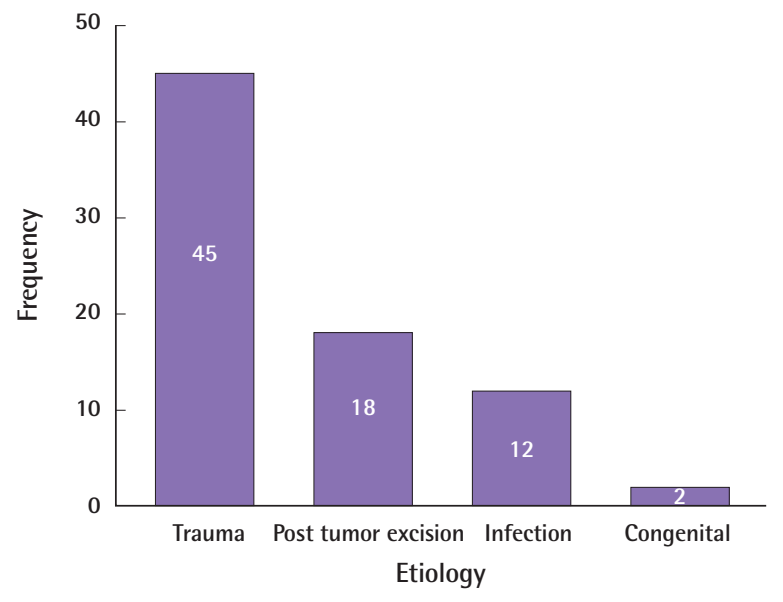

Table 2. Types of flap used according to site of defect

\begin{tabular}{|c|c|c|c|c|c|c|c|c|c|c|c|c|c|}
\hline Flap & Nose & Eyelid & Cheek & Lip & PMR & SMR & Orbit & TR & $\mathrm{AR}$ & IOR & Palate & Chin & Total \\
\hline Forehead & 12 & 9 & 9 & 18 & 3 & - & - & - & - & - & - & - & 51 \\
\hline Nasolabial & 5 & - & 1 & 2 & - & - & - & - & - & - & - & - & 8 \\
\hline Temporalis muscle & - & - & 1 & 1 & - & - & 1 & - & - & - & - & - & 3 \\
\hline Deltopectoral & - & - & - & 1 & - & 1 & - & 1 & - & - & - & 1 & 4 \\
\hline Cervical & - & - & - & - & - & - & - & - & 1 & - & - & - & 1 \\
\hline Platysma & - & - & 2 & - & - & - & - & - & - & - & - & 1 & 3 \\
\hline Sternomastoid & - & - & 2 & - & - & 1 & - & - & - & - & - & - & 3 \\
\hline Arm & - & - & - & - & - & - & - & - & - & 1 & - & - & 1 \\
\hline Bernard-Von Burow & - & - & - & 1 & - & - & - & - & - & - & - & - & 1 \\
\hline Tongue & - & - & - & - & - & - & - & - & - & - & 1 & 1 & 2 \\
\hline Post-auricular & - & - & - & - & - & - & - & - & 4 & - & - & - & 4 \\
\hline Cheek & - & - & - & 1 & - & - & - & - & - & - & - & - & 1 \\
\hline Forearm & - & - & 1 & - & - & - & - & - & - & - & - & - & 1 \\
\hline Abbe & - & - & - & 1 & - & - & - & - & - & - & - & - & 1 \\
\hline Estlander & - & - & - & 1 & - & - & - & - & - & - & - & - & 1 \\
\hline Abbe-Estlander & - & - & - & 1 & - & - & - & - & - & - & - & - & 1 \\
\hline Total & 17 & 9 & 16 & 27 & 3 & 2 & 1 & 1 & 5 & 1 & 1 & 3 & 86 \\
\hline
\end{tabular}


Table 3. Complications following use of locoregional flaps

\begin{tabular}{|lc|}
\hline Complications & Number (\%) \\
\hline Donor site infection & $5(22.7)$ \\
Total flap failure & $3(13.6)$ \\
Tumor recurrence in recipient site & $3(13.6)$ \\
Tumor occurrence in donor site & $1(4.5)$ \\
Nasal aperture narrowing & $1(4.5)$ \\
Recipient site infection & $6(27.3)$ \\
Lack of oral seal & $1(4.5)$ \\
Lip deviation & $1(4.5)$ \\
Flap contracture & $1(4.5)$ \\
Total & $22(100.0)$ \\
\hline
\end{tabular}

had immediate reconstruction. All immediate reconstructions were in patients with post-tumor excision defects.

The timing of flap division ranged from 19 to 120 days with a mean of $38.0 \pm 19.8$ days. There was no statistically significant relationship between the type of flap raised and timing of flap division $(\mathrm{P}=0.350)$. Similarly, there was no statistically significant relationship between patient age and timing of flap division $(\mathrm{P}=0.550)$. Duration of hospital admission ranged between 8 to 246 days with a mean of $51.9 \pm 39.6$ days. Complications following the use of locoregional flaps were recorded in 22 patients and included tumor recurrences in the recipient sites, 3 (3.9\%) cases, tumor occurrence in the donor site, $1(1.3 \%)$ case, and infection, 11 (14.3\%) cases (Table 3). There was no statistically significant relationship between age of patients and complications $(\mathrm{P}=0.334)$.

\section{DISCUSSION}

Different types of locoregional flaps have been used in the reconstruction of soft tissue defects in the OMFR and these include forehead, nasolabial, submental, deltopectoral, and pectoralis major flaps [11]. Generally, the choice of locoregional flap is determined by the site, size, and shape of the defect, past medical history (such as previous surgeries and radiotherapy), presence of significant scarring at the pedicle base, age and sex of patient, patient choice, and surgeon skill/preference among other factors.

In this study, more males than females had oral and maxillofacial soft tissue reconstruction and this may be related to etiological factors. Trauma and tumor excision accounted for most of the defects, and studies on trauma and tumors involving the OMFR in this environment have shown a male preponderance $[13,14]$. This is similar to findings from other studies [15]. However, a female preponderance has also been reported [16]. Sex incidence is influenced by variables such as social, cultural, and religious factors. A greater percentage of the populations in the area of this study are Muslims, and as a result of the enforcement of the Islamic sharia system, women in this part of the state live a largely indoor life. This may also account for the male preponderance noted in this review.

The age of the patients ranged from 4 to 76 years and this reflects the varying age groups in which locoregional flaps can be successfully used. Although microvascular free flaps have been used in children, their smaller vascular pedicle size (and the feasibility of good anastomosis) and possible long-term functional deficit remain some of the important challenges [17]. These difficulties can be overcome with locoregional flaps in some cases. About 12 (15.6\%) of the cases reconstructed in this study were in the pediatric age group.

The forehead flap was the most common type of locoregional flap used. The choice of forehead flap over other flaps such as the pectoralis major or deltopectoral in this study may be related to the site and size of the defects that were managed. Generally, cheek defects can be reconstructed using primary closure, full thickness skin graft, rhomboid flap, bilobed flap, cervicofacial advancement flap, temporoparietal fascia flap, temporalis muscle flap, submental flap, deltopectoral flap, forehead flap, free radial forearm flap, and free anterolateral thigh flap. Lip defects can be reconstructed using primary closure, Abbe flap, AbbeEstlander flap, Estlander flap, Gillis flap, Karapandzic flap, Webster crescentic advancement flap, Webster-Bernard flap, step flap, Schuchardt flap, nasolabial, Fujimori gate flap, total free radial forearm flap, and lateral arm flap $[11,18]$. However, in our experience, the forehead flap provides adequate tissue (for both external cover and internal oral lining) that can be used to cover defects as far as the lower border of the mandible (Fig. 2); hence its choice for defects in the cheek and lip, which constituted more than half of the cases noted in this study. The long reach of the forehead flap to as low as the upper cervical region has been documented [19]. The nasolabial and myomucosal flaps were the preferred options for lip reconstruction in other studies [20]. The late presentation of cases with tumor and orofacial gangrene in our environment results in extensive soft tissue defects requiring a locoregional flap that can offer adequate bulk and color match, which is readily provided by the forehead flap. In addition, the forehead flap is easy to mobilize when compared to other locoregional flaps such as the latissimus dorsi and pectoralis major. The cervical flap is a suitable alternative for the reconstruction of large defects of the lower face (Fig. 3). The frequent use of forehead and nasolabial flaps in nasal reconstruction in this study is consistent with other studies $[11,16]$. The nasolabial flap is ideal for small to medium-sized defects.

Immediate reconstruction of tissue defects has the advantages of ensuring a reduced number of surgical procedures; the pro- 
Fig. 2. A case of forehead flap reconstruction

A patient with alveolar soft part sarcoma of the cervicofacial region and who had immediate forehead flap reconstruction of the defect following tumor excision. (A) Frontal and (B) lateral preoperative view. (C) Immediate reconstruction of the defect with a forehead flap. (D) 10 days after surgery.
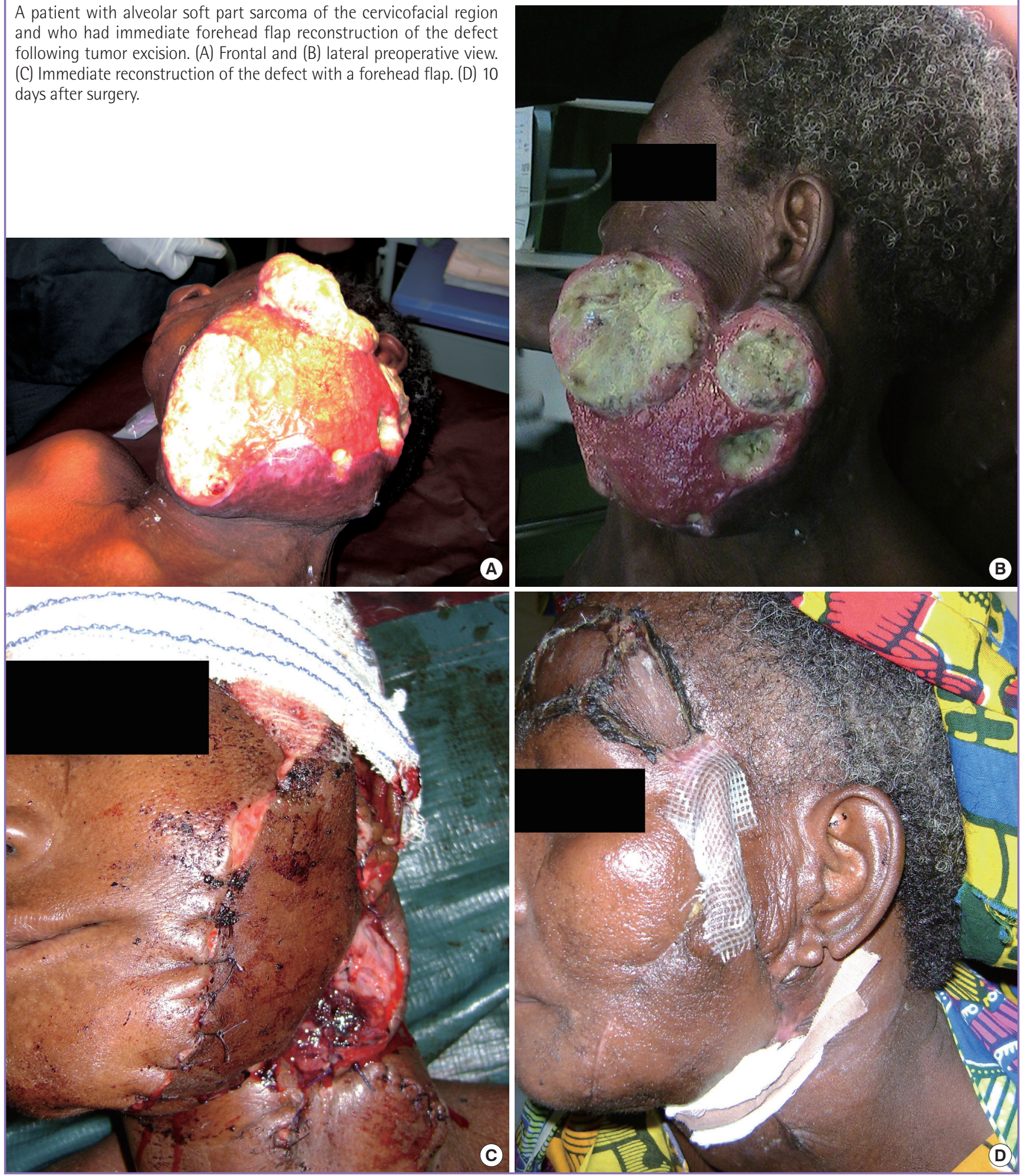

tection and preservation of vital structures; shorter hospital stay; reduced economic cost of treatment; rapid oral rehabilitation; return to normal social life; early administration of postoperative radiotherapy; and minimal fibrosis and soft tissue con- traction $[21,22]$. In this study, only 10 patients $(15.2 \%)$ had immediate reconstruction. This may be related to etiological factors and financial constraints. Trauma (mainly road traffic crash) and infection (cancrum oris) accounted for $73.7 \%$ of the orofa- 


\section{Fig. 3. A case of cervical flap reconstruction}

A patient who presented with soft tissue sarcoma of the cheek and underwent immediate reconstruction using an anteriorly based cervical flap following tumor excision. (A) Cheek defect following tumor excision. (B) Immediate reconstruction using a cervical flap. (C) Skin grafting of donor site in the neck.
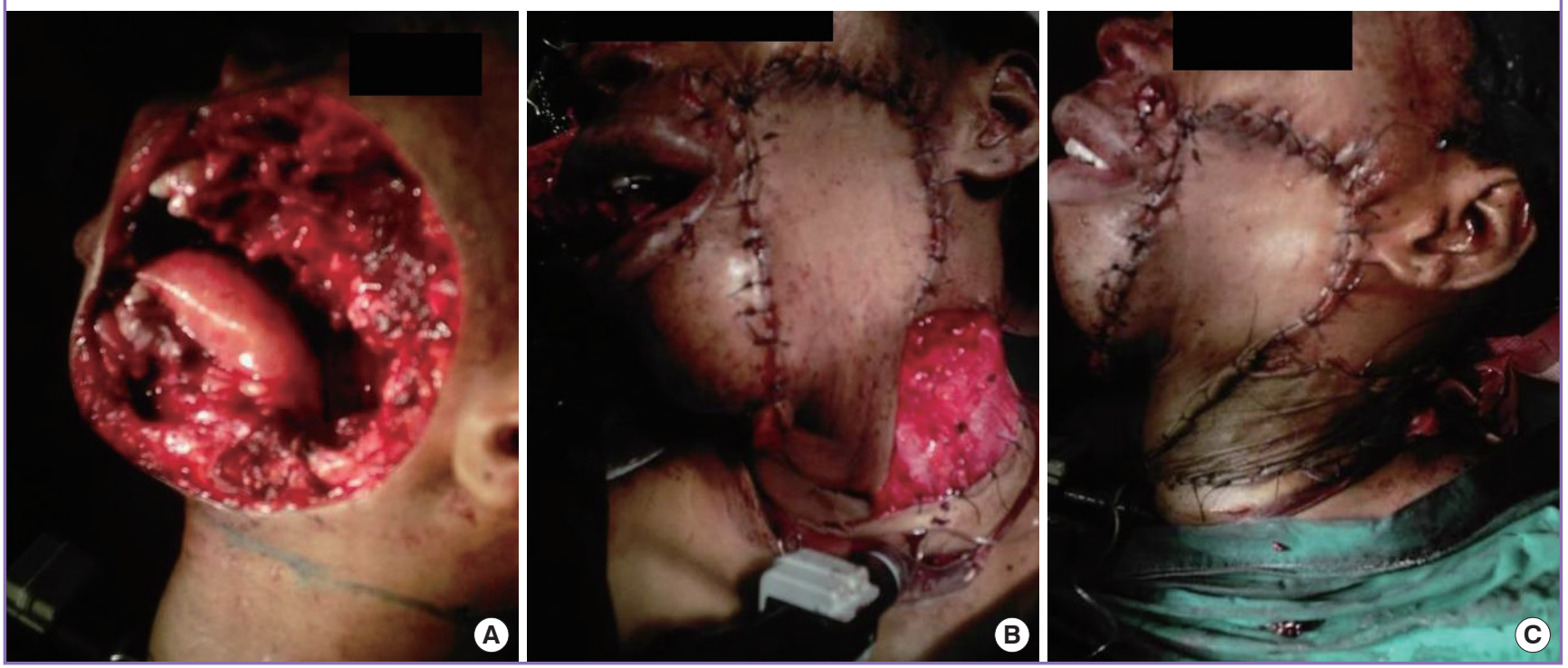

cial soft tissue defects in this retrospective analysis and are usually associated with heavily contaminated wounds that require meticulous wound care before reconstruction can be undertaken. Similarly, in the active phase of cancrum oris, a waiting time is usually required to allow the gangrenous tissues to be well demarcated from normal tissues. Financial constraints also impacted on the ability of some patients to pay for their treatment early, and this was made more difficult by the poor implementation of the National Health Insurance Scheme (NHIS). Despite the introduction of the NHIS, which is meant to ensure that every patient has access to good health services, patients still face challenges with their treatment. Problems associated with the implementation of the NHIS include poverty (unemployed individuals are unable to meet the requirements of a pre-payment plan), cultural beliefs, dilapidated health infrastructure, ill-defined criteria for referral, and the narrow range of diseases accepted within the scheme [23].

Complication following defect reconstruction was noted in 22 (28.6\%) patients and postoperative donor or recipient site infection was the most common. Others were total flap failure, tumor recurrence in the recipient bed, and tumor occurrence in the donor site. In contrast to our findings, other studies reported no infective complications $[15,16]$. The high $(14.3 \%)$ incidence of infection noted in this study may be related to poor wound dressing technique, and nutritional deficiencies (most of the patients were of lower socio-economic status). In addition, due to limited space, our patients are nursed in large open wards accommodating patients with diverse surgical conditions; this may further increase the risk of wound contamination. Tumor recurrence at the recipient bed in the orofacial region post-reconstruction is well documented in the literature $[24,25]$. The absence of frozen section technique in this environment, compared to other environments, limits our ability to achieve tumorfree margins prior to reconstruction. Early detection of recurrences allows for excision of the tumor and reconstruction with a second flap. However, in our environment, patient follow-up remains a significant challenge due to poverty, long distance to a hospital, unavailability of telecommunication services in some areas, and the perception of wellness by patients. Some of these factors should be considered in deciding whether to undertake an immediate or delayed reconstruction in malignant tumor cases.

Although a range of reconstructive options are available for functional and aesthetic restoration of defects in the OMFR, locoregional flaps provide excellent tissues for orofacial reconstruction and remain an important option, especially where free tissue transfer is not feasible. The type of locoregional flap used should be carefully selected based on patient and defect characteristics to reduce or avoid some of the complications associated with their use.

\section{REFERENCES}

1. De Sousa A. Psychological issues in acquired facial trauma. Indian J Plast Surg 2010;43:200-5.

2. Janis JE, Kwon RK, Attinger CE. The new reconstructive 
ladder: modifications to the traditional model. Plast Reconstr Surg 2011;127:205S-12S.

3. Huband M. Prosthetic rehabilitation. Dermatol Clin 2011; 29:325-30.

4. Ruka S, Kazuo K. Skin graft. Plast Surg Int 2012;2012: 563493.

5. Wysocki AB D-MW. Enhance your knowledge of skin grafts. OR Nurs 2008;2:30-8.

6. Cunha MS, Nakamoto HA, Herson MR, et al. Tissue expander complications in plastic surgery: a 10-year experience. Rev Hosp Clin Fac Med Sao Paulo 2002;57:93-7.

7. Cho JY, Jang YC, Hur GY, et al. One stage reconstruction of skull exposed by burn injury using a tissue expansion technique. Arch Plast Surg 2012;39:118-23.

8. Chim H, Salgado CJ, Seselgyte R, et al. Principles of head and neck reconstruction: an algorithm to guide flap selection. Semin Plast Surg 2010;24:148-54.

9. Hunt JP, Buchmann LO. The supraclavicular artery flap for lateral skull and scalp defects: effective and efficient alternative to free tissue transfer. J Neurol Surg Rep 2014;75:e5-e10.

10. Colletti G, Autelitano L, Tewfik K, et al. Autonomized flaps in secondary head and neck reconstructions. Acta Otorhinolaryngol Ital 2012;32:329-35.

11. Baliarsing AS, Thorat TS, Gupta A. Flap selection in head and neck cancer reconstruction. Int J Otorhinolaryngol Clin 2013; 5:63-76.

12. Uchendu OJ. Challenges of practicing histopathology in a developing country: the Nigerian perspective. Ann Biomed Sci 2013;12:68-74.

13. Obuekwe ON, Ojo MA, Akpata O, et al. Maxillofacial trauma due to road traffic accidents in Benin City, Nigeria: a prospective study. Ann Afr Med 2003;2:58-63.

14. Adebayo ET, Ajike SO, Adebola A, et al. Oral and Maxillo- facial soft tissue sarcomas in an Africa population. Niger J Surg 2006;8:135-9.

15. Chan RC, Chan JY. Deltopectoral flap in the era of microsurgery. Surg Res Pract 2014;2014:420892.

16. Rao J, Deora H. Surgical excision with forehead flap as single modality treatment for Basal cell cancer of central face: single institutional experience of 50 cases. J Skin Cancer 2014; 2014:320792.

17. Weizman N, Gil Z, Wasserzug O, et al. Surgical ablation and free flap reconstruction in children with malignant head and neck tumors. Skull Base 2011;21:165-70.

18. Pletcher SD, Kim DW. Current concepts in cheek reconstruction. Facial Plast Surg Clin North Am 2005;13:267-81.

19. Strauch B, Vasconez LO, Hall-Findlay EJ, et al. Grabb's encyclopedia of flaps. Boston: Little, Brown; 1990.

20. Siqueira EJ, Alvarez GS, Laitano FF, et al. Lower lip reconstruction strategies. Rev Bras Cir Plast 2012;27:536-41.

21. Martin PJ, O’Leary MJ, Hayden RE. Free tissue transfer in oromandibular reconstruction: necessity or extravagance? Otolaryngol Clin North Am 1994;27:1141-50.

22. Kroll SS, Schusterman MA, Reece GP. Costs and complications in mandibular reconstruction. Ann Plast Surg 1992;29: 341-7.

23. Metiboba S. Nigeria's National Health Insurance Scheme: the need for beneficiary participation. Res J Int Stud 2011; 22:51-6.

24. Knoetgen J 3rd, Choudry U, Finical SJ, et al. Head and neck reconstruction with a second free flap following resection of a recurrent malignancy. Ann Plast Surg 2005;55:378-83.

25. Pickford MA, Soutar DS. Intraoral reconstruction using a second free flap for recurrent or metachronous carcinoma. Br J Plast Surg 1995;48:559-63. 